\title{
On the concept of random orientation in far-field electromagnetic scattering by nonspherical particles
}

\author{
Michael I. Mishchenko ${ }^{1, *}$ And Maxim A. YuRKIN²,3 \\ ${ }^{1}$ NASA Goddard Institute for Space Studies, 2880 Broadway, New York, NY 10025, USA \\ ${ }^{2}$ Voevodsky Institute of Chemical Kinetics and Combustion, SB RAS, Institutskaya str. 3, 630090 Novosibirsk, Russia \\ ${ }^{3}$ Novosibirsk State University, Pirogova 2, 630090 Novosibirsk, Russia \\ *Corresponding author: michael.i.mishchenko@nasa.gov
}

Received XX Month XXXX; revised XX Month, XXXX; accepted XX Month XXXX; posted XX Month XXXX (Doc. ID XXXXX); published XX Month XXXX

\begin{abstract}
Although the model of randomly oriented nonspherical particles has been used in a great variety of applications of far-field electromagnetic scattering, it has never been defined in strict mathematical terms. In this Letter we use the formalism of Euler rigid-body rotations to clarify the concept of statistically random particle orientations and derive its immediate corollaries in the form of most general mathematical properties of the orientationaveraged extinction and scattering matrices. Our results serve to provide a rigorous mathematical foundation for numerous publications in which the notion of randomly oriented particles and its light-scattering implications have been considered intuitively obvious. (C) 2016 Optical Society of America
\end{abstract}

OCIS codes: (290.5850) Scattering, particles; (290.5825) Scattering theory (030.5620); Radiative transfer; (290.5855) Scattering, polarization.

http://dx.doi.org/10.1364/OL.99.099999

The concept of randomly oriented nonspherical particles has been widely used in studies of far-field electromagnetic scattering and radiative transfer (see, e.g., Refs. [1-20] and references therein). Yet the notion of random orientation has always been assumed to be self-explanatory, and its consequences such as optical isotropy of the extinction matrix and the dependence of the scattering matrix on only the scattering angle have been taken for granted as physically obvious and needing no proof. Furthermore, a specific integral over the three Eulerian angles has been assumed-again without explicit justification-to represent the desired averaging over random particle orientations [4,7-12,14-19,21,22].

One should keep in mind however that literature on electromagnetic scattering by particles contains many examples of "physical obviousness" turning out to be a shaky and misleading argument. Therefore, the great practical importance of the model of electromagnetic scattering by randomly oriented particles makes it essential to replace the presumed physical obviousness of the main consequences of this model by explicit mathematical proofs. We do that in the rest of this Letter.

Although the notion of a randomly oriented particle may appear to be intuitively evident, it needs to be formulated and parameterized mathematically before it can be used to derive useful corollaries. First of all, it is imperative to recognize that the randomness of particle orientations can only be achieved in the statistical sense, i.e., by assuming that (i) an optical observable is measured over a sufficiently long period of time, and (ii) the scattering process is ergodic $[14,19]$. These assumptions allow one to replace time averaging by ensemble averaging, including averaging over particle orientations. In this paper we apply the concept of random orientations to the far-field extinction and scattering matrices. As such, it is relevant to scattering by a single random particle and a small and sparse random multiparticle group as well as to the radiative transfer theory. For simplicity, we will consider only far-field scattering by a single random particle or a random two-particle group, but generalization to other relevant situations is straightforward $[14,19]$.

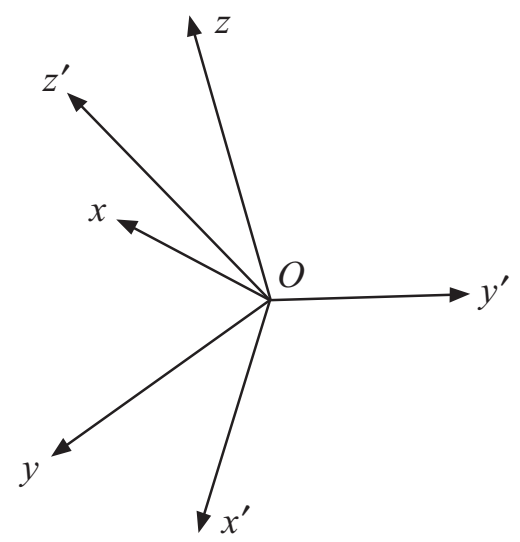

Fig. 1. The laboratory, $O x y z$, and particle, $O x^{\prime} y^{\prime} z^{\prime}$, reference frames having a common origin.

Second of all, it is quite natural to parameterize the orientation of a particle with respect to a fixed right-handed reference frame $O x y z$ by affixing a right-handed reference frame $O x^{\prime} y^{\prime} z^{\prime}$ to the 
particle and specifying the orientation of $O x^{\prime} y^{\prime} z^{\prime}$ relative to $O x y z$. Note that the two reference frames are assumed to have the same origin representing, e.g., the center of mass of the particle (Fig. 1).

It has been known since the classical work by Leonhard Euler that the orientation of $O x^{\prime} y^{\prime} z^{\prime}$ with respect to $O x y z$ can be parameterized uniquely (except when $\beta= \pm \pi$ ) by the set of three rotation angles $g=\{\alpha, \beta, \gamma\}$, as shown in Fig. 2. It is therefore natural to parameterize the orientation of the particle by specifying the three Euler angles that transform the laboratory reference frame $O x y z$ into the particle reference frame $O x^{\prime} y^{\prime} z^{\prime}$. A statistical distribution of particle orientations can then be parameterized by the normalized probability density function $P(g)$ such that

$$
\int_{0}^{2 \pi} \mathrm{d} \alpha \int_{0}^{\pi} \mathrm{d} \beta \int_{0}^{2 \pi} \mathrm{d} \gamma P(g)=1
$$

The average of a function $f(g)$ over particle orientations is then calculated according to

$$
\langle f\rangle=\int_{0}^{2 \pi} \mathrm{d} \alpha \int_{0}^{\pi} \mathrm{d} \beta \int_{0}^{2 \pi} \mathrm{d} \gamma f(g) P(g) .
$$

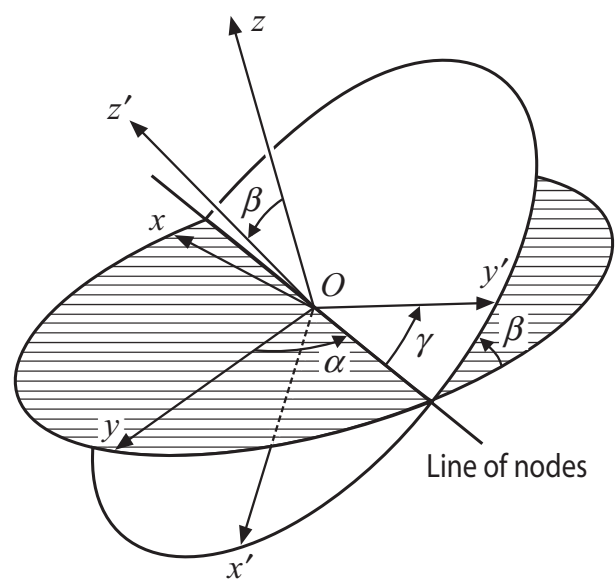

Fig. 2. The Euler rotation angles $0 \leq \alpha<2 \pi, 0 \leq \beta \leq \pi$, and $0 \leq \gamma<2 \pi$ as defined in Ref. [23].

The next step is to determine the form of the probability density function $P_{\mathrm{r}}(g)$ corresponding to the random (or uniform) orientation distribution. Let us affix to a nonspherical particle two different particle reference frames, $O x^{\prime} y^{\prime} z^{\prime}$ and $O x^{\prime \prime} y^{\prime \prime} z^{\prime \prime}$, having the same origin (Fig. 3). We will determine $P_{\mathrm{r}}(g)$ by requiring that irrespective of the particle morphology and of the choice of the two particle reference frames, averaging any function of particle orientation over the uniform orientation distribution of either $O x^{\prime} y^{\prime} z^{\prime}$ or $O x^{\prime \prime} y^{\prime \prime} z^{\prime \prime}$ yields exactly the same result. This natural definition is an essential step from a physical notion of random orientation to rigorous mathematical implications.

Let $\tilde{g}=\{\tilde{\alpha}, \beta, \tilde{\gamma}\}$ be the rotation transforming $O x^{\prime \prime} y^{\prime \prime} z^{\prime \prime}$ into $O x^{\prime} y^{\prime} z^{\prime}$ and $g \widetilde{g}$ be the rotation resulting from the rotation $\widetilde{g}$ followed by the rotation $g=\{\alpha, \beta, \gamma\}$. Then the above definition of randomness of particle orientations implies the identity

$$
\begin{aligned}
\int_{0}^{2 \pi} \mathrm{d} \alpha \int_{0}^{\pi} \mathrm{d} \beta \int_{0}^{2 \pi} \mathrm{d} \gamma f(g) P_{\mathrm{r}}(g) \\
\quad=\int_{0}^{2 \pi} \mathrm{d} \alpha \int_{0}^{\pi} \mathrm{d} \beta \int_{0}^{2 \pi} \mathrm{d} \gamma f(g \widetilde{g}) P_{\mathrm{r}}(g)
\end{aligned}
$$

valid for any fixed $\widetilde{g}$. From the standpoint of the group theory, $P_{\mathrm{r}}(g)$ corresponds to a normalized right Haar measure on the 3D rotation group. This measure is by definition invariant to a right translation by an arbitrary group element. The left Haar measure is defined by replacing $g \widetilde{g}$ with $\widetilde{g} g$ on the right-hand side of Eq. (3).

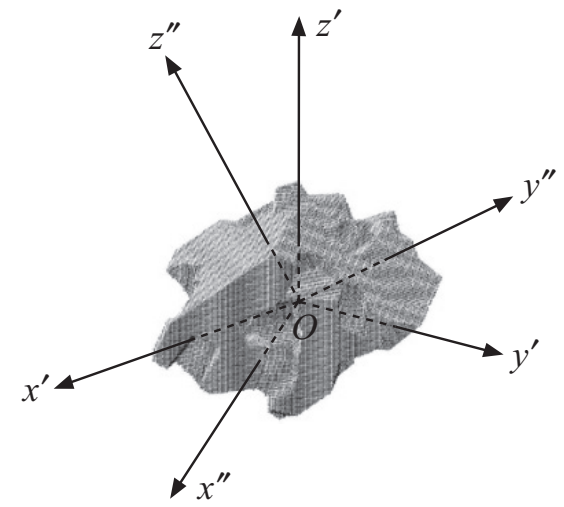

Fig. 3. Two particle reference frames having a common origin.

The normalized right Haar measure is unique for any parametrization of the group [24,25]. In particular, a fundamental consequence of Eq. (3) derived in \$1.3 of Chapter 1 in Ref. [26] and $\S 1.6$ of Chapter 1 in Ref. [27] (see also Ref. [28]) is that

$$
P_{\mathrm{r}}(g)=\frac{\sin \beta}{8 \pi^{2}} \text {. }
$$

Moreover, the left and right Haar measures coincide for the 3D rotation group $[27,28]$ (which implies that Eq. (3) remains valid with the same $P_{\mathrm{r}}(g)$ upon replacing $g \widetilde{g}$ with $\widetilde{g} g$ ), so we do not distinguish between them further on. The derivation of Eq. (4) was originally performed using the zxz-notation of the Euler angles (named by the axes of consecutive rotations). However, it is also valid for any other (proper) notation in which the first and the last axes coincide, including the zyz notation of Fig. 2. By contrast, the Haar measure is more complicated for Tait-Bryan angles wherein all three rotation axes are different [28]. This justifies the conventional choice of the Euler angles for the calculation of orientation-averaged optical properties.

Thus we can conclude that averaging over random particle orientations is represented by

$\langle f\rangle_{\mathrm{r}}=\frac{1}{8 \pi^{2}} \int_{0}^{2 \pi} \mathrm{d} \alpha \int_{0}^{\pi} \mathrm{d} \beta \sin \beta \int_{0}^{2 \pi} \mathrm{d} \gamma f(\alpha, \beta, \gamma)$.

Let us now consider the most fundamental properties of the $4 \times 4$ extinction matrix averaged over random orientations of a nonspherical particle. The definition of the extinction matrix $\mathbf{K}(\hat{\mathbf{n}})$ can be found in Section 2.7 of Ref. [12], Section 3.8 of Ref. [14] or Section 13.3 of Ref. [19]. Let $\hat{\mathbf{n}}$ and $\hat{\mathbf{n}}_{1}$ be the unitvectors specifying two different directions of propagation of the incident plane electromagnetic wave and let the corresponding extinction matrices be defined relative to the laboratory reference frame $O x y z$ and the auxiliary reference frame $O x_{1} y_{1} z_{1}$, respectively (Fig. 4). Note that $\hat{\mathbf{n}}$ points in the positive direction of the $z$-axis, $\hat{\mathbf{n}}_{1}$ points in the positive direction of the $z_{1}$-axis, and the planes $O x z$ and $O x_{1} z_{1}$ serve as the reference planes for defining the corresponding sets of the four Stokes parameters [12,14,19]. The rotations $g$ and $\widetilde{g}$ transform the laboratory reference frame 
$O x y z$ into $O x^{\prime} y^{\prime} z^{\prime}$ and $O x_{1} y_{1} z_{1}$, respectively.

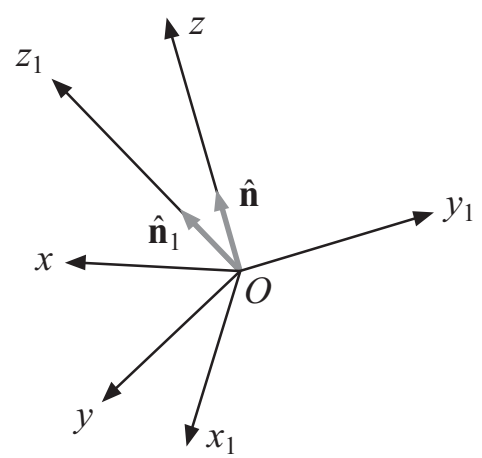

Fig. 4. The laboratory, $O x y z$, and auxiliary, $O x_{1} y_{1} z_{1}$, reference frames.

The implication of these definitions is as follows:

$\mathbf{K}\left(\hat{\mathbf{n}}_{1} ; \tilde{g}\right)=\mathbf{K}(\hat{\mathbf{n}} ; e) \Rightarrow \mathbf{K}\left(\hat{\mathbf{n}}_{1} ; g \widetilde{g}\right)=\mathbf{K}(\hat{\mathbf{n}} ; g)$,

where the second argument denotes the particle orientation and $e$ is the identity element of the rotation group (i.e., no rotation). In other words, simultaneous rotation of the incident beam and the particle does not change the extinction matrix. Then Eqs. (3) and (6) imply

$\left\langle\mathbf{K}\left(\hat{\mathbf{n}}_{1} ; g\right)\right\rangle_{\mathrm{r}}=\langle\mathbf{K}(\hat{\mathbf{n}} ; g)\rangle_{\mathrm{r}} \equiv \mathbf{K}$,

where the averaging is defined by Eq. (5). In other words, the orientation-averaged extinction matrix is independent of the direction of propagation of the incident plane wave and of the choice of the reference plane used to define the Stokes parameters.

The derivation of the general structure of $\mathbf{K}$ is based on the consideration of pairs of reciprocal scattering configurations as defined in Ref. [2], i.e., those with the incident and scattering directions interchanged and inverted. The standard assumption has always been that for each particle orientation there is another one, called reciprocal. However, this assumption in Sec. 5.22 of Ref. [2] was not accompanied by an explicit mathematical definition of statistically random orientations, which left it unclear whether the numbers of original and reciprocal orientations are always equal. Therefore, we instead exploit Eq. (3) and the fact that the reciprocal configuration can be obtained from the original one by a single rotation $\widetilde{g}$. The particular form of this rotation is known for arbitrary $\hat{\mathbf{n}}$ and $\hat{\mathbf{n}}^{\prime}$ [2] but is unimportant for the following. Equation (3) then yields

$$
\langle\mathbf{K}(\hat{\mathbf{n}} ; g \tilde{g})\rangle_{\mathrm{r}}=\langle\mathbf{K}(\hat{\mathbf{n}} ; g)\rangle_{\mathrm{r}}=\frac{1}{2}\langle\mathbf{K}(\hat{\mathbf{n}} ; g \tilde{g})+\mathbf{K}(\hat{\mathbf{n}} ; g)\rangle_{\mathrm{r}} .
$$

This formula allows for the application of the reciprocity relation for the amplitude scattering matrix [29], which leads to the cancellation of the elements $K_{13}, K_{31}, K_{24}$, and $K_{42}$. Furthermore, owing to Eq. (7), the extinction matrix must be invariant with respect to an arbitrary rotation of the reference plane around $\hat{\mathbf{n}}$ : $\mathbf{L}(\eta) \mathbf{K} \mathbf{L}(-\eta) \equiv \mathbf{K}$, where $\mathbf{L}(\eta)$ is the Stokes rotation matrix for the rotation angle $\eta[12,14,19]$. It is then straightforward to show that the resulting orientation-averaged extinction matrix has a highly symmetric structure given by

$$
\mathbf{K}=\left[\begin{array}{cccc}
K_{11} & 0 & 0 & K_{14} \\
0 & K_{11} & K_{23} & 0 \\
0 & -K_{23} & K_{11} & 0 \\
K_{14} & 0 & 0 & K_{11}
\end{array}\right]
$$

and has only three independent elements.

In addition, let us assume that the nonspherical particle is accompanied by its mirror counterpart. The following derivation requires the second particle to be a mirror image of the first particle with respect to the fixed scattering plane Oxz (assumption 2 in Sec. 5.22 of Ref. [2]). We set the particle reference frames such that they form a mirror-symmetric configuration with respect to the $O x z$ plane in the default orientation. We then note that the reflection $h$ with respect to the $O x z$ plane is a combination of the rotation $g_{y}$ around the $y$-axis by an angle $\pi$ followed by the inversion $i$ $\left(h=i g_{y}\right)$, and $i$ commutes with any rotation or reflection [30]. Then, the orientation average of any property of the mirror particle is given by

$$
\begin{aligned}
\left\langle f_{h}(g)\right\rangle_{\mathrm{r}} & \doteq\langle f(g h)\rangle_{\mathrm{r}}=\left\langle f_{i}\left(g g_{y}\right)\right\rangle_{\mathrm{r}}=\left\langle f_{i}(g)\right\rangle_{\mathrm{r}}=\left\langle f_{i}\left(g_{y} g\right)\right\rangle_{\mathrm{r}} \\
& =\langle f(h g)\rangle_{\mathrm{r}},
\end{aligned}
$$

where $f_{i}(g) \doteq f(g i)$, and we have used Eq. (3) as well as its counterpart with $g$ and $\tilde{g}$ interchanged. In other words Eq. (10) proves that reflections and rotations effectively commute inside the orientation averaging. In application to $\mathbf{K}$ this implies that

$$
\left\langle\mathbf{K}_{h}(\hat{\mathbf{n}} ; g)\right\rangle_{\mathrm{r}}+\langle\mathbf{K}(\hat{\mathbf{n}} ; g)\rangle_{\mathrm{r}}=\langle\mathbf{K}(\hat{\mathbf{n}} ; h g)+\mathbf{K}(\hat{\mathbf{n}} ; g)\rangle_{\mathrm{r}},
$$

i.e., the averaging is performed on pairs for which the amplitude scattering matrices are intimately related [2], thereby causing the cancellation of the remaining off-diagonal elements of $\mathbf{K}$. The latter is then diagonal and has only one independent element:

$\mathbf{K}=C_{\mathrm{ext}} \operatorname{diag}[1,1,1,1]$,

where $C_{\text {ext }}$ is the extinction cross section. It is easily seen that Eq. (12) is also valid for a single randomly oriented particle with a plane of symmetry.

Finally, let us consider the most general properties of the orientation-averaged $4 \times 4$ Stokes scattering matrix. We remind the reader that the scattering matrix $\mathbf{F}\left(\hat{\mathbf{n}}^{\prime}, \hat{\mathbf{n}}\right)$ for the incidence direction $\hat{\mathbf{n}}$ and the scattering direction $\hat{\mathbf{n}}^{\prime}$ relates the Stokes parameters of the incident plane wave and the scattered spherical wave in the far zone of the particle. In this case both sets of the Stokes parameters are defined with respect to the scattering plane (i.e., the plane through $\hat{\mathbf{n}}$ and $\hat{\mathbf{n}}^{\prime}$ ).

As before, let $\hat{\mathbf{n}}$ and $\hat{\mathbf{n}}_{1}$ be the unit vectors specifying two different incidence directions and let the corresponding scattering planes be given by the $O x z$ and $O x_{1} z_{1}$ planes of the reference frames $O x y z$ and $O x_{1} y_{1} z_{1}$, respectively (Fig. 4). The corresponding scattering directions, $\hat{\mathbf{n}}^{\prime}$ and $\hat{\mathbf{n}}_{1}^{\prime}$, are obtained by rotating the vectors $\hat{\mathbf{n}}$ and $\hat{\mathbf{n}}_{1}$ clockwise around the $y$ - and $y_{1}$ axis, respectively, through the same angle $\Theta$ called the scattering angle.

Completely analogous to the case of the extinction matrix, simple geometrical considerations suggest that

$\mathbf{F}\left(\hat{\mathbf{n}}_{1}^{\prime}, \hat{\mathbf{n}}_{1} ; g \widetilde{g}\right)=\mathbf{F}\left(\hat{\mathbf{n}}^{\prime}, \hat{\mathbf{n}} ; g\right)$,

which, together with Eq. (3), implies that 
$\left\langle\mathbf{F}\left(\hat{\mathbf{n}}_{1}^{\prime}, \hat{\mathbf{n}}_{1} ; g\right)\right\rangle_{\mathrm{r}} \equiv\left\langle\mathbf{F}\left(\hat{\mathbf{n}}^{\prime}, \hat{\mathbf{n}} ; g\right)\right\rangle_{\mathrm{r}} \equiv \mathbf{F}(\Theta)$,

where the averaging is defined by Eq. (5). Thus, the orientationaveraged scattering matrix is independent of the incidence direction and of the orientation of the scattering plane and is a function of the scattering angle only.

One can then use the reciprocity relation for the amplitude scattering matrix [29] and the line of thought in Section 5.22 of Ref. [2] to show that the orientation-averaged scattering matrix has 10 independent elements and is given by

$$
\mathbf{F}(\Theta)=\left[\begin{array}{cccc}
F_{11}(\Theta) & F_{12}(\Theta) & F_{13}(\Theta) & F_{14}(\Theta) \\
F_{12}(\Theta) & F_{22}(\Theta) & F_{23}(\Theta) & F_{24}(\Theta) \\
-F_{13}(\Theta) & -F_{23}(\Theta) & F_{33}(\Theta) & F_{34}(\Theta) \\
F_{14}(\Theta) & F_{24}(\Theta) & -F_{34}(\Theta) & F_{44}(\Theta)
\end{array}\right] .
$$

The only essential missing step in Ref. [2] is the explicit use of Eq. (8) with $\mathbf{K}(\hat{\mathbf{n}} ; g)$ being replaced by $\mathbf{F}\left(\hat{\mathbf{n}}^{\prime}, \hat{\mathbf{n}} ; g\right)$.

Assuming also that the randomly oriented nonspherical particle is accompanied by its randomly oriented mirror counterpart and using Eq. (10) or the corresponding analogue of Eq. (11) (with reflection defined with respect to the scattering plane) ultimately yields the conventional block-diagonal scattering matrix:

$$
\mathbf{F}(\Theta)=\left[\begin{array}{cccc}
F_{11}(\Theta) & F_{12}(\Theta) & 0 & 0 \\
F_{12}(\Theta) & F_{22}(\Theta) & 0 & 0 \\
0 & 0 & F_{33}(\Theta) & F_{34}(\Theta) \\
0 & 0 & -F_{34}(\Theta) & F_{44}(\Theta)
\end{array}\right]
$$

(cf. Refs. [1,2]). It can easily be shown that this formula also applies to a single randomly oriented particle with a plane of symmetry.

The previous discussion has been based on the assumption that all rotations of a particle occur in such a way that a point inside the particle (e.g., its center of mass) remains fixed at the origin of the laboratory reference frame. However, the extinction and scattering matrices are invariant with respect to parallel translations of the particle as long as these translations are sufficiently small (see Section 3.1 of Ref. [12] or Section 13.9 of Ref. [19]). Therefore, all properties of the orientation-averaged extinction and scattering matrices remain the same if the particle wobbles arbitrarily during the measurement.

In summary, we began this Letter by giving the explicit mathematical definition of a randomly oriented (in the statistical sense) nonspherical particle. We then derived the main properties of the far-field extinction and scattering matrices as direct mathematical corollaries of Eq. (3) rather than assumed them to be intuitively obvious. As a result, we have provided the rigorous (and long overdue) justification of the main premises implicit in Refs. [120] and numerous related publications.

Funding. National Aeronautics and Space Administration (NASA) Remote Sensing Theory Program, NASA ACE Project; Russian Science Foundation (RSF) grant No. 14-15-00155.

Acknowledgment We appreciate instructive discussions with Joop Hovenier, Andrey Mishchenko, Katharina Sander, and Larry Travis as well as two constructive anonymous reviews.
1. F. Perrin, J. Chem. Phys. 10, 415-427 (1942).

2. H. C. van de Hulst, Light Scattering by Small Particles (Wiley, 1957)

3. C. F. Bohren and D. R. Huffman, Absorption and Scattering of Light by Small Particles (Wiley, 1983).

4. M. K. Singham, S. B. Singham, and G. C. Salzman, J. Chem. Phys. 85, 3807-3815 (1986).

5. W. J. Wiscombe and A. Mugnai, Single Scattering from Nonspherical Chebyshev Particles: A Compendium of Calculations (NASA, 1986).

6. P. W. Barber and S. C. Hill, Light Scattering by Particles: Computational Methods (World Scientific, 1990).

7. M. I. Mishchenko, J. Opt. Soc. Am. A 8, 871-882 (1991).

8. N. G. Khlebtsov, Appl. Opt. 31, 5359-5365 (1992).

9. A. Z. Dolginov, Yu. N. Gnedin, and N. A. Silant'ev, Propagation and Polarization of Radiation in Cosmic Media (Gordon and Breach, 1995).

10. D. W. Mackowski and M. I. Mishchenko, J. Opt. Soc. Am. A 13, 2266-2278 (1996).

11. M. I. Mishchenko, J. W. Hovenier, and L. D. Travis, Light Scattering by Nonspherical Particles: Theory, Measurements, and Applications (Academic, 2000).

12. M. I. Mishchenko, L. D. Travis, and A. A. Lacis, Scattering, Absorption, and Emission of Light by Small Particles (Cambridge University, 2002), http://www.giss.nasa.gov/staff/mmishchenko/books.html.

13. J. W. Hovenier, C. van der Mee, and H. Domke, Transfer of Polarized Light in Planetary Atmospheres: Basic Concepts and Practical Methods (Kluwer, 2004).

14. M. I. Mishchenko, L. D. Travis, and A. A. Lacis, Multiple Scattering of Light by Particles: Radiative Transfer and Coherent Backscattering (Cambridge University, 2006), http://www.giss.nasa.gov/staff/mmishchenko/books.html.

15. A. Doicu, T. Wriedt, and Y. A. Eremin, Light Scattering by Systems of Particles (Springer, 2006).

16. F. Borghese, P. Denti, and R. Saija, Scattering from Model Nonspherical Particles: Theory and Applications to Environmental Physics (Springer, 2007).

17. D. W. Mackowski and M. I. Mishchenko, J. Quant. Spectrosc. Radiat. Transfer 112, 2182-2192 (2011).

18. M. A. Yurkin and A. G. Hoekstra, J. Quant. Spectrosc. Radiat. Transfer 112, 2234-2247 (2011).

19. M. I. Mishchenko, Electromagnetic Scattering by Particles and Particle Groups: An Introduction (Cambridge University, 2014).

20. K.-N. Liou and P. Yang, Light Scattering by Ice Crystals: Fundamentals and Applications (Cambridge University, 2016).

21. V. V. Varadan and V. K. Varadan, Phys. Rev. D 21, 388-394 (1980).

22. L. Tsang, J. A. Kong, and R. T. Shin, Theory of Microwave Remote Sensing (Wiley, 1985).

23. D. A. Varshalovich, A. N. Moskalev, and V. K. Khersonskii, Quantum Theory of Angular Momentum (World Scientific, 1988). Original Russian edition: Nauka, 1975.

24. A. Weil, L'intégration dans les Groupes Topologiques et ses Applications (Hermann, 1940).

25. L. Nachbin, The Haar Integral (Van Nostrand, 1965).

26. I. M. Gel'fand, R. A. Minlos, and Z. Ya. Shapiro, Representations of the Rotation and Lorentz Groups and their Applications (Pergamon, 1963). Original Russian edition: Fizmatlit, 1958.

27. M. A. Naimark, Linear Representations of the Lorentz Group (Pergamon, 1964). Original Russian edition: Fizmatlit, 1958.

28. R. E. Miles, Biometrika 52, 636-639 (1965).

29. D. S. Saxon, Phys. Rev. 100, 1771-1775 (1955).

30. M. Tinkham, Group Theory and Quantum Mechanics (McGraw-Hill, 1964).

\section{References}




\section{References}

1. F. Perrin, "Polarization of light scattered by isotropic opalescent media," J. Chem. Phys. 10, 415-427 (1942).

2. H. C. van de Hulst, Light Scattering by Small Particles (Wiley, 1957).

3. C. F. Bohren and D. R. Huffman, Absorption and Scattering of Light by Small Particles (Wiley, 1983).

4. M. K. Singham, S. B. Singham, and G. C. Salzman, "The scattering matrix for randomly oriented particles," J. Chem. Phys. 85, 38073815 (1986).

5. W. J. Wiscombe and A. Mugnai, Single Scattering from Nonspherical Chebyshev Particles: A Compendium of Calculations (NASA, 1986).

6. P. W. Barber and S. C. Hill, Light Scattering by Particles: Computational Methods (World Scientific, 1990).

7. M. I. Mishchenko, "Light scattering by randomly oriented axially symmetric particles," J. Opt. Soc. Am. A 8, 871-882 (1991).

8. N. G. Khlebtsov, "Orientational averaging of light-scattering observables in the T-matrix approach," Appl. Opt. 31, 5359-5365 (1992).

9. A. Z. Dolginov, Yu. N. Gnedin, and N. A. Silant'ev, Propagation and Polarization of Radiation in Cosmic Media (Gordon and Breach, 1995).

10. D. W. Mackowski and M. I. Mishchenko, "Calculation of the $T$ matrix and the scattering matrix for ensembles of spheres," J. Opt. Soc. Am. A 13, 2266-2278 (1996).

11. M. I. Mishchenko, J. W. Hovenier, and L. D. Travis, Light Scattering by Nonspherical Particles: Theory, Measurements, and Applications (Academic, 2000).

12. M. I. Mishchenko, L. D. Travis, and A. A. Lacis, Scattering, Absorption, and Emission of Light by Small Particles (Cambridge University, 2002), http://www.giss.nasa.gov/staff/mmishchenko/books.html.

13. J. W. Hovenier, C. van der Mee, and H. Domke, Transfer of Polarized Light in Planetary Atmospheres: Basic Concepts and Practical Methods (Kluwer, 2004).

14. M. I. Mishchenko, L. D. Travis, and A. A. Lacis, Multiple Scattering of Light by Particles: Radiative Transfer and Coherent Backscattering (Cambridge University, 2006), http://www.giss.nasa.gov/staff/mmishchenko/books.html.

15. A. Doicu, T. Wriedt, and Y. A. Eremin, Light Scattering by Systems of Particles (Springer, 2006).

16. F. Borghese, P. Denti, and R. Saija, Scattering from Model Nonspherical Particles: Theory and Applications to Environmental Physics (Springer, 2007).

17. D. W. Mackowski and M. I. Mishchenko, "A multiple sphere $T$ matrix Fortran code for use on parallel computer clusters," J. Quant. Spectrosc. Radiat. Transfer 112, 2182-2192 (2011).

18. M. A. Yurkin and A. G. Hoekstra, "The discrete-dipoleapproximation code ADDA: capabilities and known limitations," J. Quant. Spectrosc. Radiat. Transfer 112, 2234-2247 (2011).

19. M. I. Mishchenko, Electromagnetic Scattering by Particles and Particle Groups: An Introduction (Cambridge University, 2014).

20. K.-N. Liou and P. Yang, Light Scattering by Ice Crystals: Fundamentals and Applications (Cambridge University, 2016).

21. V. V. Varadan and V. K. Varadan, "Multiple scattering of electromagnetic waves by randomly distributed and oriented dielectric scatterers," Phys. Rev. D 21, 388-394 (1980).

22. L. Tsang, J. A. Kong, and R. T. Shin, Theory of Microwave Remote Sensing (Wiley, 1985).

23. D. A. Varshalovich, A. N. Moskalev, and V. K. Khersonskii, Quantum Theory of Angular Momentum (World Scientific, 1988). Original Russian edition: Nauka, 1975.

24. A. Weil, L'intégration dans les Groupes Topologiques et ses Applications (Hermann, 1940).

25. L. Nachbin, The Haar Integral (Van Nostrand, 1965).

26. I. M. Gel'fand, R. A. Minlos, and Z. Ya. Shapiro, Representations of the Rotation and Lorentz Groups and their Applications (Pergamon, 1963). Original Russian edition: Fizmatlit, 1958.

27. M. A. Naimark, Linear Representations of the Lorentz Group (Pergamon, 1964). Original Russian edition: Fizmatlit, 1958.

28. R. E. Miles, “On random rotations in $R^{3}$," Biometrika 52, 636-639 (1965).

29. D. S. Saxon, "Tensor scattering matrix for the electromagnetic field," Phys. Rev. 100, 1771-1775 (1955).

30. M. Tinkham, Group Theory and Quantum Mechanics (McGraw-Hill, 1964). 